\title{
Angular momentum sharing in dissipative collisions
}

\author{
G. Casini, G. Poggi, M. Bini, S. Calamai, P. Maurenzig, A. Olmi, G. Pasquali, A. \\ A. Stefanini, N. Taccetti \\ Istituto Nazionale di Fisica Nucleare and Università di Firenze, I-50125 Florence, Italy \\ J. C. Steckmeyer, R. Laforest* \\ Laboratoire de Physique Corpusculaire, IN2P3-CNRS, ISMRA et Université, F-14050 \\ Caen-Cedex, France \\ F. Saint-Laurentit \\ GANIL, BP 5027, 14021 Caen-Cedex, France
}

(June 29, 2021)

\begin{abstract}
Light charged particles emitted by the projectile-like fragment were measured in the direct and reverse collision of ${ }^{93} \mathrm{Nb}$ and ${ }^{116} \mathrm{Sn}$ at $25 \mathrm{AMeV}$. The experimental multiplicities of Hydrogen and Helium particles as a function of the primary mass of the emitting fragment show evidence for a correlation with net mass transfer. The ratio of Hydrogen and Helium multiplicities points to a dependence of the angular momentum sharing on the net mass transfer.
\end{abstract}

25.70.-z, 25.70.Lm, 25.70.Pq 
It is now experimentally established that binary or quasi-binary dissipative processes continue to dominate the heavy-ion reaction cross-section well into the intermediate energy regime [1] [7. In this context, a still open field of investigation concerns the degree of equilibrium attained in the internal degrees of freedom and, in particular, the partition of energy [8] and angular momentum between the two reaction partners.

At low bombarding energies $(\leq 15 \mathrm{AMeV})$, several experimental findings (concerning mass and charge drift, variances, excitation energies of reaction products) are rather well accounted for, in some cases also quantitatively, by models based on the stochastic exchange of single nucleons (see, e.g., [9]). At larger bombarding energies, the relevance of such a mechanism becomes somewhat uncertain, due to the decrease of interaction times, to the increasing importance of the reaction dynamics and to associated non-equilibrium effects.

In the ${ }^{120} \mathrm{Sn}+{ }^{100} \mathrm{Mo}$ collision at $19.1 \mathrm{AMeV}$ the fission probability $P_{\text {fiss }}$ of the projectileand target-like fragment (PLF and TLF) was measured 10 as a function of the primary mass $A$. For a given $A$, corresponding to different net mass transfers for PLF and TLF, $P_{\text {fiss }}$ was found to be significantly larger for the TLF (which gained mass), even at large TKEL (Total Kinetic Energy Loss). The observed effect is a clear signature of the lack of an overall equilibrium between the two partners at the end of the interaction.

In the ${ }^{100} \mathrm{Mo}+{ }^{120} \mathrm{Sn}$ collision at $14.1 \mathrm{AMeV}$ [1] a similar behavior was found also in the binary exit channel, where the highly excited fragments de-excite mainly by light particle emission. The observed correlation between the total number of emitted nucleons and the net mass transfer indicates a non-equilibrium excitation energy partition between the reaction products, with an excess of excitation being deposited in the fragment which gains nucleons. Similar conclusions had been drawn by other authors 12 16, but remained quite controversial. Although the existence of such correlations is compatible, by itself, with a nucleon exchange picture, the fact that they are largely independent of the degree of inelasticity 11.,15] is difficult to understand within the present versions of the stochastic nucleon exchange model and deserves new investigation.

Up to now no study has been performed concerning possible correlations between net mass transfer and angular momentum sharing. This letter presents for the first time a direct evidence for a correlation between angular momentum sharing and net mass transfer.

Beams of ${ }^{93} \mathrm{Nb}$ and ${ }^{116} \mathrm{Sn}$ at $24.9 \mathrm{AMeV}$ were delivered by the GANIL accelerator with an excellent time resolution (about 550 ps and 350 ps FWHM for Nb and Sn, respectively). The system ${ }^{93} \mathrm{Nb}+{ }^{116} \mathrm{Sn}$ was studied in direct and reverse kinematics, as already done in a previous experiment at lower energy [11]. This method allows to obtain information on both partners of the collision, although the detection is optimized for the PLF and its associated particles. It is equivalent to studying, in two separate runs, the PLF and TLF of the $\mathrm{Nb}+\mathrm{Sn}$ collision. The slight mass asymmetry guarantees a common range of PLF masses in the exit channels in both kinematic cases, even at moderate TKEL. Beam and target feasibility reasons (as well as the wish to have a system near to the ${ }^{100} \mathrm{Mo}+{ }^{120} \mathrm{Sn}$ studied at $14 \mathrm{AMeV}$ [11]) guided the choice of the system. The isotope of Sn, while abundant enough to make a beam, has an $\mathrm{N} / \mathrm{Z}$ ratio similar enough to that of ${ }^{93} \mathrm{Nb}$ so as to reduce the possible role of isospin.

Heavy $(A \geq 20)$ reaction products were detected with position-sensitive gas detectors [1,2]. The FWHM resolution was $3.5 \mathrm{~mm}$ for position and $600-750 \mathrm{ps}$ for the timeof-flight (including the beam contribution). From the measured velocity vectors, primary 
(pre-evaporative) quantities were deduced event-by-event with an improved version [17] of the kinematic coincidence method. For elastic events, the FWHM resolution of the primary mass was $\approx 2$ amu. The background of incompletely measured events of higher multiplicity was estimated and subtracted from the results [17]. Behind the forward gas detectors, on one side of the beam, an array of 46 Silicon detectors (covering a sizeable region below and around the grazing angle) allowed to deduce the secondary (post-evaporative) mass of the PLF [18]. Light charged particles were measured with the scintillator array "Le Mur" [19]. It consists of 96 pads of fast plastic scintillator NE102, $2 \mathrm{~mm}$ thick (threshold $\approx 3.2 \mathrm{AMeV}$ for protons and $\alpha$-particles) and covered in an axially symmetric geometry the region from $2^{\circ}$ to $18.5^{\circ}$ behind the gas and Silicon detectors. However, because of the shadows of these detectors, not all scintillator pads could be used. More details on the experimental set-up and analysis method are given in Ref. [18].

The data presented in this letter are focused on binary events in which light charged particles were detected in the scintillators in coincidence with PLF and TLF, additionally requiring that the PLF hits a forward gas detector and one of the Silicon detectors behind it.

Results about the analysis of the average number $\Delta A$ of nucleons emitted by the PLF as a function of its primary mass $A$ are presented in detail elsewhere [18]. Here we just need to mention that $\Delta A$ versus $A$ gives origin to two distinct correlations for the $\mathrm{Nb}+\mathrm{Sn}$ and $\mathrm{Sn}+\mathrm{Nb}$ data, similarly to what already observed in the system ${ }^{100} \mathrm{Mo}+{ }^{120} \mathrm{Sn}$ at $14 \mathrm{AMeV}$ [1], which gave a direct evidence for the dependence of $\Delta A$ on the net mass transfer.

The data of "Le Mur" were used to deduce the multiplicity of light charged particles emitted by the PLF source. The limited solid angle of the scintillator array already selected mainly light particles emitted by the PLF and this geometric selection was further strengthened in the analysis by rejecting all slow particles stopped in the pads. The remaining particles were cleanly identified in charge $\mathrm{Z}$ but their isotopic composition could not be determined. Therefore in the following we will just refer to Hydrogen and Helium ions.

Because of the shadows produced by the Silicon detectors, only light particles emitted on the other side of the beam with respect to the PLF were considered in the analysis. Moreover, for a cleaner selection of the PLF source, only light particles emitted in a forward range (from about $14^{\circ}$ to $70^{\circ}$ ) in the PLF frame were considered. In this frame, the experimental lightparticle velocity spectra display an evaporation-like shape, consistent — within experimental errors — with the results of evaporation calculations obtained with the statistical code GEMINI [20]. To deduce the multiplicities of particles emitted by the PLF, Monte Carlo efficiency corrections were performed, assuming an evaporative isotropic emission. Due to these corrections the absolute values of the light particle multiplicities may be affected by uncertainties up to $\pm 30 \%$. However, the uncertainty is much smaller (of the order of $\pm 10 \%$ ) on the relative values.

The average multiplicities $\left\langle M_{H}\right\rangle$ and $\left\langle M_{H e}\right\rangle$ of Hydrogen and Helium ions were determined as a function of the primary mass $A$ of the emitting PLF, for bins of TKEL. The left and right columns of Fig. 1 present the results for Hydrogen and Helium, respectively. The circles and squares refer to the light charged particles emitted from the PLF in the ${ }^{93} \mathrm{Nb}+$ ${ }^{116} \mathrm{Sn}$ and ${ }^{116} \mathrm{Sn}+{ }^{93} \mathrm{Nb}$ reaction, respectively. Two assumptions on the excitation energy sharing were used in the Monte Carlo simulations to deduce the absolute multiplicities from the experimental ones. The solid symbols show the results obtained under the assumption 
of a non-equilibrium excitation energy sharing (dependent on the net mass transfer), as deduced from the analysis of the correlations between $\Delta A$ and $A$ [18. The open symbols are the results obtained from the same data assuming an energy sharing independent of the net mass transfer. It is apparent that the results are rather insensitive to the particular physical hypothesis on energy sharing used for the correction. The absolute multiplicities for the channel without net mass transfer are lower than the values obtained by GEMINI calculations by a factor of about 1.5-2. However, they are in fair agreement with the experimental values obtained for the PLF emission in the similar systems Mo + Mo at 23.7 $\mathrm{AMeV}$ [21] and $\mathrm{Xe}+\mathrm{Sn}$ at $25 \mathrm{AMeV}$ [22].

Concerning the dependence on the primary mass $A$ of the PLF, the multiplicities grow with increasing $A$. We want to draw attention on the fact that, for a given TKEL, the multiplicities in the symmetric exit channel are quite different in the two kinematic cases, although the mass of the emitting PLF is the same $(A \approx 105)$. They are larger in the direct reaction (where the PLF has gained mass) with respect to the reverse reaction (where it has lost an equal amount of nucleons). Being the multiplicity of light charged particles an increasing function of the excitation energy of the emitter, one can deduce that the nucleus gaining mass is more excited than the one loosing mass. This behavior is evident beyond errors at all TKEL values for the Helium data, whereas for Hydrogen it becomes weaker with increasing TKEL.

These results on the light charged particles are thus qualitatively in good agreement with those concerning the total number of evaporated nucleons, $\Delta A$, deduced on the base of a kinematic reconstruction. It is worth noting that the measurements of light charged particles in the scintillator array "Le Mur" and of heavy reaction products in the gas detectors are independent. Therefore the results of Fig. 1 should be little affected by "instrumental" correlations of the kind discussed in Ref. [23].

As the relative multiplicities are less uncertain than the absolute ones, we further concentrated on the multiplicity ratio of Hydrogen and Helium, $\left\langle M_{H}\right\rangle /\left\langle M_{H e}\right\rangle$. Figure $\mathbb{2}(\mathrm{a}$ (a) shows this ratio for the exit channel without net mass transfer. The solid dots refer to the direct reaction ${ }^{93} \mathrm{Nb}+{ }^{116} \mathrm{Sn}$ at $25 \mathrm{AMeV}$, the solid squares to the reverse reaction at the same bombarding energy. Based on the results for the average excitation energy partition [18], the excitation energy of the PLF is estimated from TKEL assuming an equal division of the total excitation energy, although all arguments that follow are rather insensitive to this hypothesis. The two sets of experimental data are very similar, as expected from the weak dependence of the light particle multiplicities on the mass of the emitter. In both cases the ratio $\left\langle M_{H}\right\rangle /\left\langle M_{H e}\right\rangle$ strongly decreases with increasing excitation energy.

It is known since long that in a statistical decay process of a moderately excited nucleus the ratio of Hydrogen to Helium decreases with increasing spin of the emitter [24 26]. Statistical-model calculations with GEMINI [20], suggest that this finding may still hold in our range of energies and masses. In Fig. 2(a) the open symbols joined by dotted lines are the results of GEMINI calculations for an excited ${ }^{93} \mathrm{Nb}$ at various values of the spin. Indeed the ratio between Hydrogen and Helium particles is found to be rather sensitive to the angular momentum of the evaporating nucleus, with large angular momenta favoring the emission of the more massive Helium particles with respect to the lighter Hydrogen isotopes. The comparison of the experimental data with the calculations suggests that the transfer of angular momentum from the orbital motion to the internal degrees of freedom of the 
colliding nuclei is weak for low TKEL (peripheral collisions) and becomes larger when going to larger TKEL values (that is, to more central collisions). However, quantitative estimates of the spin values are difficult as there are experimental indications that at large excitations GEMINI tends to underestimate the emission of intermediate mass fragments [18].

Let us concentrate for the remaining part of this letter on the events leading to nearly symmetric mass division $(A \approx 105)$ in the exit channel. Figure $2(\mathrm{~b})$ presents the ratio of light charged particles $\left\langle M_{H}\right\rangle /\left\langle M_{H e}\right\rangle$ emitted by the PLF measured in the direct and reverse kinematics for the collision ${ }^{93} \mathrm{Nb}+{ }^{116} \mathrm{Sn}$ at $25 \mathrm{AMeV}$ (solid dots and squares, respectively). Here the excitation energy $E^{*}$ of the PLF has been estimated from the measured TKEL assuming an excitation energy division in agreement with that deduced from the total number of nucleons evaporated from the fragments, but again the arguments that follow do not depend on this hypothesis.

For the PLF measured in the direct reaction - which therefore experienced a net mass gain - the experimental results (dots) correspond to rather low ratios $\left\langle M_{H}\right\rangle /\left\langle M_{H e}\right\rangle$, thus being an indication of high spin. The opposite holds for the PLF measured in the reverse reaction, which experienced a net loss of nucleons. In this second case the results (squares) correspond to larger ratios $\left\langle M_{H}\right\rangle /\left\langle M_{H e}\right\rangle$, thus pointing to lower spin of the emitter. This observation clearly indicates that the net gain of nucleons is correlated with an excess not only of excitation energy [11, 15, 18], but also of angular momentum. The same conclusion would hold true also in case of a different excitation energy partition, as a relative shift of the two sets of experimental points along the horizontal axis would leave the data for the direct reaction below those for the reverse one, thus indicating in any case an asymmetric sharing of angular momentum.

As verified with GEMINI calculations, this interpretation of the data in terms of netmass-transfer dependence of both excitation energy and angular momentum partition can explain the above mentioned weaker dependence of the Hydrogen multiplicity on the netmass-transfer at larger excitations. In fact, in the direct reaction the simultaneous increase of both excitation energy and spin gives origin to two opposite effects. The higher excitation energy of PLF tends to increase the average multiplicity of Hydrogen, but the larger spin tends to depress it. On the contrary, for Helium both the higher excitation energy and the larger spin contribute to the increase of the average multiplicity.

It is worth stressing that although the specific interpretation in terms of angular momentum sharing requires that the observed light charged particles emission is of statistical origin, in any case the experimental observation proves by itself that no full equilibrium in the relative degrees of freedom between the two final nuclei, both with $A \approx 105$, has been achieved at the end of the interaction phase. In spite of their equal masses, the two nuclei bear memory of the different ways they have been produced, either by net gain or loss of nucleons.

The present data, together with other observations - like the persistence of steep correlations between $\triangle A$ and $A$ even at large TKEL and the systematically larger than expected mass variances $\sigma_{A}^{2}$ at large TKEL [11,18] - suggest the presence of some other mechanism besides the mere stochastic exchange of single nucleons across a window in the dinuclear system.

For example, the fluctuations in the rupture of a long stretched neck might be an essential ingredient to explain the experimental features. They can explain in a natural way the 
observation of huge mass variances in spite of rather short interaction times. Asymmetric neck ruptures, with the lighter collision partner obtaining a larger share of the neck matter, could sizably contribute to the net mass transfer leading to symmetric mass division [6]. In this case the two final nuclei, although of equal mass, would strongly differ in shape and moment of inertia. This neck remnant, if re-absorbed, might be responsible for the larger share of angular momentum. Or it might contribute to the increased production of intermediate mass fragments in the region between the two collision partners, which is presently a highly debated topic.

Concluding, the collision ${ }^{93} \mathrm{Nb}+{ }^{116} \mathrm{Sn}$ at $25 \mathrm{AMeV}$ has been studied in direct and reverse kinematics. The light charged particles emitted by the PLF allow a qualitative estimation of the transferred angular momentum and give evidence of a situation of non-equilibrium existing between the two collision partners at the end of the interaction. In fact the net gain of nucleons appears to be correlated with an excess of both excitation energy and angular momentum. This experimental finding seems difficult to reconcile with existing models based on stochastic exchanges of singles nucleons and calls for a better theoretical understanding of the microscopic interaction mechanism, including other effects like, e.g., an explicit treatment of the neck degrees of freedom.

We wish to thank the GANIL staff for delivering high quality beams pulsed with very good time structure. We also thank R. Ciaranfi and M. Montecchi for their skillfulness in the development of dedicated electronic modules and P. Del Carmine and F. Maletta for their valuable support in the preparation of the experimental set-up. 


\section{REFERENCES}

* Permanent Address: Washington University, Medical School, BOX 8225510 Kingshiway St-Louis, MO 63110

$\dagger$ Permanent Address: DRFC/STEP, CEA/Cadarache, F-13108 Saint-Paul-lez-Durance Cedex.

[1] R. J. Charity et al., Z. Phys. A 341, 53 (1991).

[2] A. A. Stefanini et al., Z. Phys. A 351, 167 (1995).

[3] B. Lott et al. Phys. Rev. Lett. .68, 3141 (1992).

[4] L. Beaulieu et al. Phys. Rev. Lett. .77, 462 (1996).

[5] B. Borderie et al., Z. Phys. A 357, 7 (1997).

[6] Y. Larochelle et al., Phys. Rev. C 57, R1027 (1998).

[7] J. Lukasik et al., Phys. Rev. C 55, 1906 (1997).

[8] J. Tõke and W. U. Schröder, Ann. Rev. Nucl. Part. Sci. 42, 401 (1992).

[9] J. Randrup, Nucl. Phys. A 307, 319 (1978); 327, 490 (1979); 383, 468 (1982).

[10] G. Casini et al., Phys. Rev. Lett. 67, 3364 (1991).

[11] G. Casini et al., Phys. Rev. Lett. 78, 878(1997).

[12] D. R. Benton et al., Phys. Rev. C 38, 1207 (1988).

[13] J. Wilczynski et al., Phys. Lett. B 220, 497 (1989).

[14] K. Kwiatkowski et al., Phys. Rev. C 41, 958 (1990).

[15] J. Tõke et al., Phys. Rev. C 44, 390 (1991).

[16] L. Fiore et al., Phys. Rev. C 50, 1709 (1994).

[17] G. Casini et al., Nucl. Instr. Methods A 277, 445 (1989).

[18] G. Casini et al. to be published.

[19] G. Bizard et al. Nucl. Instr. Methods A 244, 483 (1986).

[20] R. J. Charity et al., Nucl. Phys. A 483, 371 (1988); 511, 59 (1990).

[21] M. Gnirs, PhD thesis, Heidelberg University, 1991.

[22] E. Genouin-Duhamel, PhD thesis, Caen University, 1999.

[23] J. Tõke et al., Nucl. Instr. Meth. A 288, 406 (1990).

[24] T. D. Thomas, Nucl. Phys. 53, 538 (1964).

[25] J. Gilat and J. R. Gover, Phys. Rev. C 3, 734 (1971).

[26] G. L. Catchen et al., Phys. Rev. C 21, 940 (1980). 


\section{FIGURES}

FIG. 1. Efficiency corrected experimental multiplicities for Hydrogen (left column) and Helium particles (right column) emitted by the PLF, plotted as a function of the primary mass $A$ of the PLF, for $100 \mathrm{MeV}$ wide bins of TKEL. The circles and squares refer to light charged particles emitted from the PLF in the ${ }^{93} \mathrm{Nb}+{ }^{116} \mathrm{Sn}$ and ${ }^{116} \mathrm{Sn}+{ }^{93} \mathrm{Nb}$ reaction, respectively. The solid and open symbols show the experimental data corrected assuming an excitation energy sharing dependent on net mass transfer (as deduced from $\Delta A$ of the fragments) and independent of it, respectively. The lines are guides to the eye. The error bars show the statistical errors.

FIG. 2. (a) Ratio of the experimental average multiplicities of Hydrogen and Helium particles, $\left\langle M_{H}\right\rangle /\left\langle M_{H e}\right\rangle$, emitted by the PLF in exit channels without net mass transfer. The solid dots (squares) refer to PLF from the direct (reverse) collision ${ }^{93} \mathrm{Nb}+{ }^{116} \mathrm{Sn}$ at $25 \mathrm{AMeV}$ and are plotted as a function of the excitation energy estimated from the data. For comparison also the

results of GEMINI calculations for several values of the spin of an emitting ${ }^{93} \mathrm{Nb}$-source are shown. The lines are drawn to guide the eye. (b) Same presentation as in part (a) except that the data refer to events leading to a symmetric mass division $(A \approx 105)$ in the exit channel. 


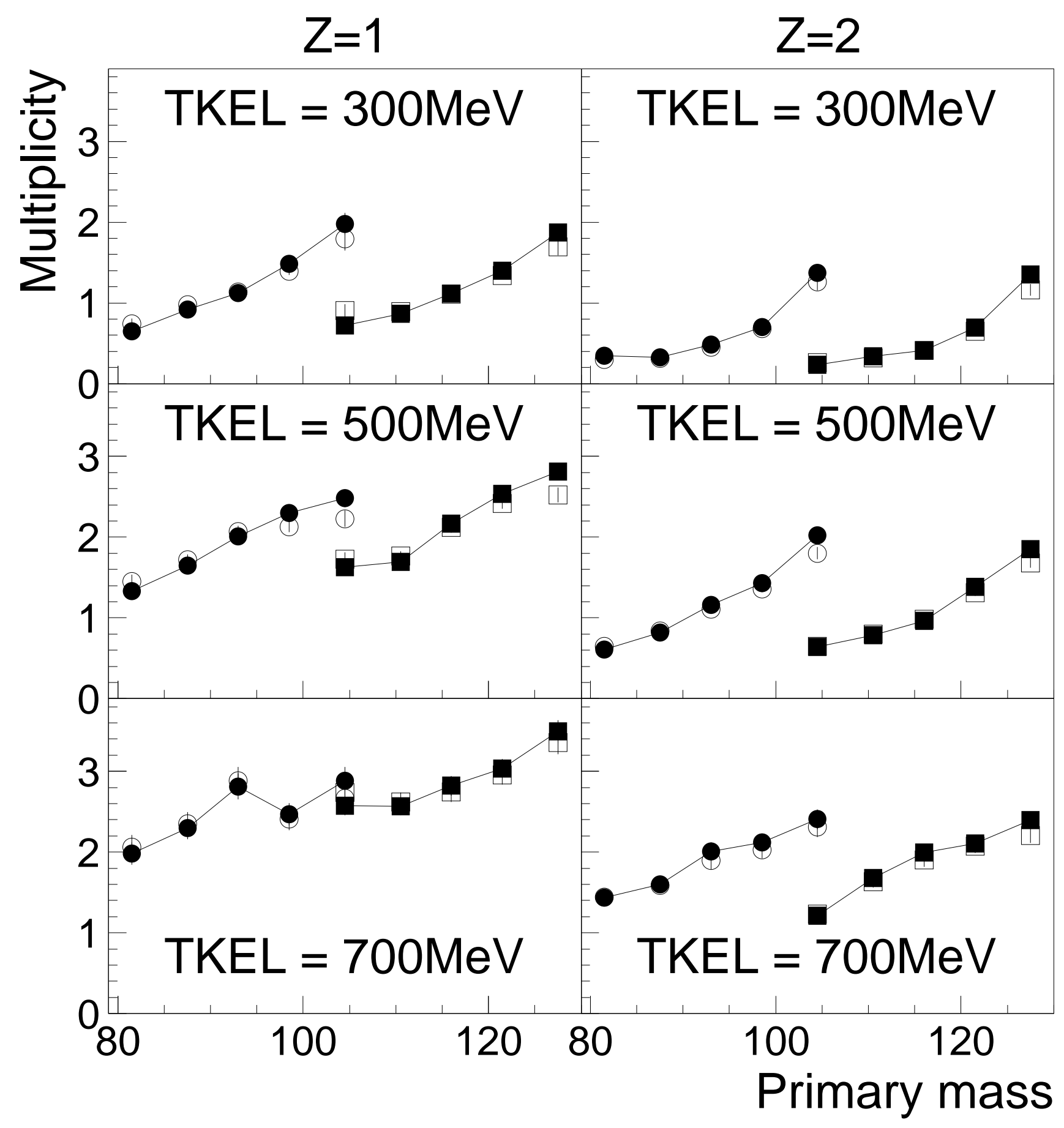




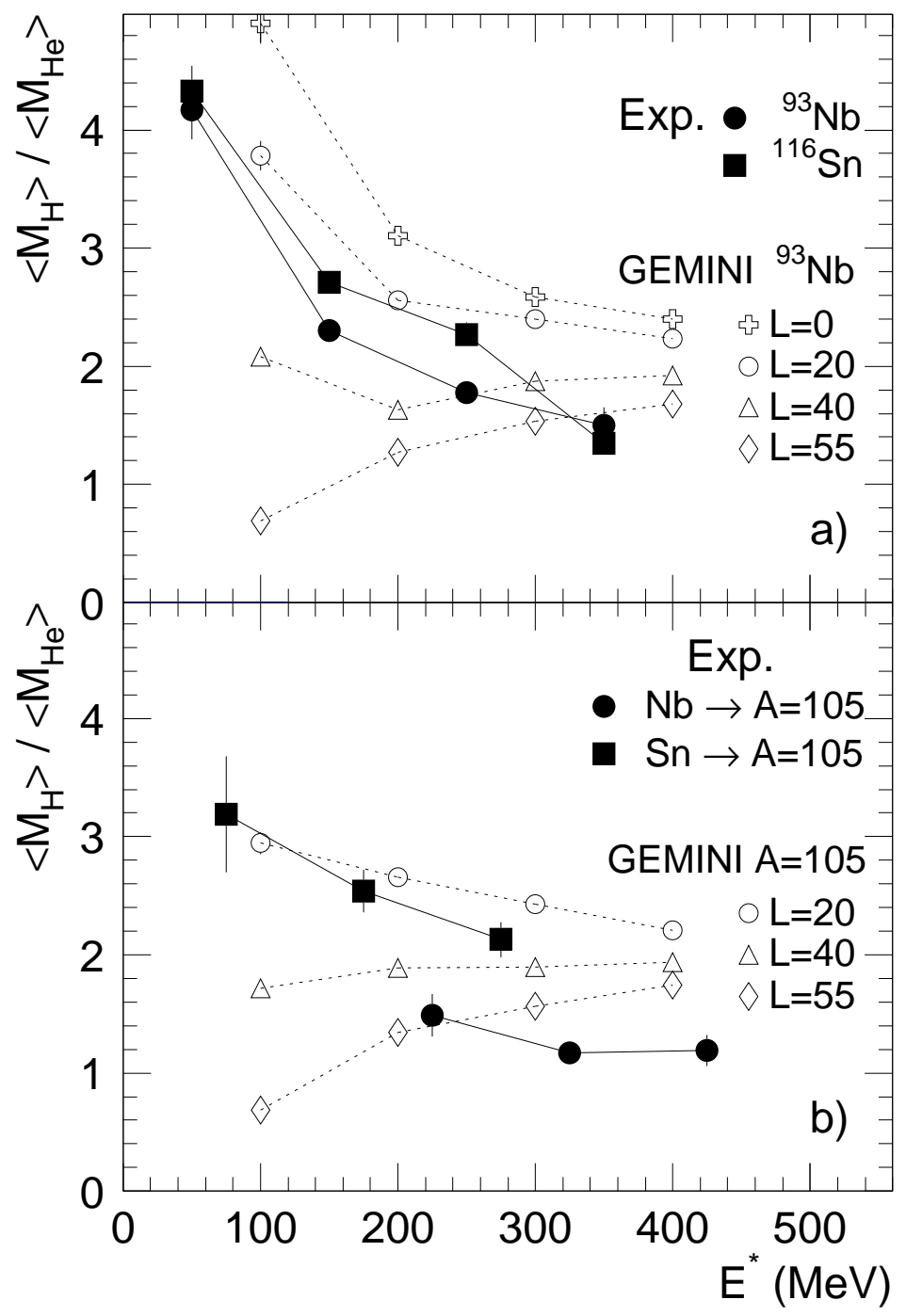

